\title{
Desenvolvimento da Cultura de Mandioca sob Interferência de Plantas Daninhas ${ }^{1}$
}

\author{
Weed Interference in the Development of the Cassava Plant
}

\author{
ALBUQUERQUE, J.A.A. ${ }^{2}$, SEDIYAMA, T. ${ }^{3}$, SILVA, A.A. ${ }^{3}$, ALVES, J.M.A. ${ }^{2}$, FINOTO, E.L. ${ }^{4}$, \\ NETO, F.A. ${ }^{5}$ e SILVA, G.R. ${ }^{6}$
}

\begin{abstract}
RESUMO - Um dos fatores que afetam o desenvolvimento da planta da mandioca é o controle inadequado das plantas daninhas. Objetivou-se com este trabalho avaliar o desenvolvimento da cultura de mandioca sob interferência de plantas daninhas. Foram realizados dois experimentos: no primeiro, os tratamentos foram compostos por períodos de convivência da cultura da mandioca com as plantas daninhas por 25, 50, 75, 100 e 125 dias após plantio e, no segundo, as plantas de mandioca permaneceram livres das plantas daninhas pelos mesmos períodos preestabelecidos. Avaliações de altura de plantas e diâmetro de caule foram realizadas aos $75,100,125,150,175,200,225,250,275,300,325$ e 350 dias após o plantio. Nas colheitas dos experimentos, realizadas aos 330 dias após plantio, avaliou-se o número e o diâmetro das raizes por planta. A convivência por intervalo igual ou superior a 50 dias após o plantio reduziu o diâmetro de caule e a altura das plantas de mandioca, assim como o número e comprimento de raízes tuberosas. O controle das plantas daninhas nessa cultura deve ser iniciado em torno de 25 dias após o plantio.
\end{abstract}

Palavras-chave: controle de plantas daninhas, macaxeira, Manihot esculenta.

ABSTRACT - One of the factors affecting the development of the cassava plant is the inadequate control of weeds. Thus, this work aimed to evaluate the development of the cassava plant under weed interference during different coexistence periods. Two experiments were installed in adjacent areas, in Viçosa, Minas Gerais - Brazil. In the first experiment, the treatments consisted of different periods of coexistence between the cassava culture and the weeds (25, 50, 75, 100 and 125 days after planting). In the second experiment, the cassava plants remained weed-free for the same pre-established periods. For both experiments, the $1.0 \times 0.5 \mathrm{~m}$ spacing was adopted, being the useful portion area constituted by the two central lines, with $1.0 \mathrm{~m}$ being left at each extremity as front borders, adding $8.0 \mathrm{~m}^{2}$ up. Plant height and stem diameter were evaluated twelve times after planting at: 75, 100, 125, 150, 175, 200, 225, 250, 275, 300, 325 and 350 days. Plant number, root length and diameter evaluations were carried out 12 months after planting. Coexistence equal or superior to 50 day interval after planting caused drastic reduction in the stem diameter and height of the cassava plants. Weed control in cassava crop should be initiated around 25 days after planting.

Keywords: weed control, cassava, Manihot esculenta.

\section{INTRODUÇÃO}

A mandioca é de grande importância para a região tropical, onde se constitui numa das principais fontes de carboidratos disponiveis aos estratos sociais de baixa renda. Sua parte mais importante, economicamente, são as raízes tuberosas, ricas em amido, utilizadas na alimentação humana e animal ou como matéria-prima para diversos derivados

1 Recebido para publicação em 14.2.2011 e aprovado em 19.7.2011.

2 Professores, Doutores, Dep. de Fitotecnia, Universidade Federal Rural de Roraima - UFRR, Boa Vista, Roraima, <anchietaufrr@gmail.com.br>; ${ }^{3}$ Professores, Doutores, Dep. de Fitotecnia, Universidade Federal de Viçosa - DFT/UFV, Bolsistas do CNPq; 36570-000 Viçosa-MG; ${ }^{4}$ Pesquisador, Doutor, Agência Paulista de Tecnologia dos Agronegócios -APTA(PRDTA-Centro Norte) Pindorama, São Paulo; ${ }^{5}$ Professor, Doutor, Curso de Agronomia, Universidade Federal do Piauí - UFPI, Bom Jesus-PI; ${ }^{5}$ Mestrando, DFT/UFV. 
industriais. Tem importante participação na geração de emprego e de renda, especialmente para pequenos e médios produtores (Albuquerque et al., 2008). No Brasil, a cultura da mandioca ocupa áreas situadas desde o norte até o sul, tanto na faixa litorânea como nas regiões mais ocidentais.

Amplamente cultivada na África tropical, Ásia e América Latina, a mandioca é a quarta cultura mais importante do mundo em desenvolvimento, sendo o alimento principal de quase um bilhão de pessoas em 105 países, onde essa raiz provê até um terço das calorias diárias (FAO, 2010).

Por se tratar de uma planta de crescimento inicial lento e que deixa o solo descoberto, a mandioca tem seu desenvolvimento influenciado pela presença de plantas daninhas, que competem pelos fatores de produção (água, luz, nutrientes e gás carbônico), ocasionalmente trazendo maiores perdas do que aquelas provocadas pelas pragas e doenças (Azevêdo et al., 2000). Todavia, os efeitos negativos observados no crescimento e desenvolvimento de uma cultura, devido à presença das plantas daninhas, não podem ser atribuídos exclusivamente à competição imposta por estas últimas. Pitelli (1985) afirma que esses efeitos são resultantes de pressões ambientais atuando diretamente em competição, alelopatia, interferência na colheita e/ou indiretamente, hospedando pragas, moléstias e nematoides.

Apesar de ser importante fonte de alimento para as regiões tropicais, em especial para o Brasil, a mandioca tem sido relativamente pouco estudada em nosso país. Nesse sentido, é necessário aumentar as pesquisas sobre a cultura da mandioca, para que se possa melhor compreender as alterações fisiológicas e bioquímicas, sendo importante avaliar a produção e distribuição dos carboidratos ao longo do seu crescimento e desenvolvimento, na presença e na ausência de plantas daninhas. Isso permitirá identificar seus reais efeitos, além da melhor compreensão das possiveis alterações verificadas na formação e nas dimensões das raízes tuberosas, em função da adoção de determinada prática cultural ou do próprio efeito das condições ambientais (Sangoi \& Kruse, 1993).

Objetivou-se com este trabalho avaliar o desenvolvimento da cultura de mandioca sob interferência de plantas daninhas.

\section{MATERIAL E MÉTODOS}

Dois experimentos foram realizados no município de Viçosa-MG, em áreas adjacentes, no campo experimental do Departamento de Fitotecnia da Universidade Federal de Viçosa, em solo classificado como Argissolo VermelhoAmarelo, cujas principais características fisicas e químicas são apresentadas na Tabela 1. Na Figura 1 encontram-se os dados referentes a precipitação, umidade relativa e temperatura média do ar no período de realização do experimento.

O delineamento experimental adotado em ambos os experimentos foi o de blocos casualizados, com sete tratamentos e quatro repetições. No primeiro experimento, os tratamentos foram compostos por períodos de convivência das plantas daninhas com a cultura da mandioca (Tabela 2). No segundo, os tratamentos corresponderam às épocas de efetivo controle das plantas daninhas a partir do plantio da cultura (Tabela 3). A área experimental foi adubada de acordo com análise do solo e conforme a recomendação para a cultura da mandioca (CFSEMG, 1999). Foram utilizadas manivas do cultivar Cacauzinha, do grupo das mandiocas-mansas, plantadas em 22/12/ 2003 no espaçamento de $1,0 \mathrm{~m}$ entre fileiras por $0,5 \mathrm{~m}$ entre plantas. A colheita dos experimentos foi realizada em 21/12/2004.

As determinações da altura de plantas e do diâmetro do caule foram realizadas em 12 épocas após o plantio: $75,100,125,150$, 175, 200, 225, 250, 275, 300, 325 e 350 dias. Para isso, foram avaliadas quatro plantas por parcela, as quais foram previamente etiquetadas. Utilizou-se uma régua de madeira graduada para medir a altura de plantas e um paquímetro para o diâmetro de caule. Aos 350 dias após o plantio, foram avaliados o número de raízes tuberosas por planta, o comprimento e o diâmetro. Para avaliação do número de raízes tuberosas por planta, foram coletadas oito plantas por parcela, sendo em seguida feita a contagem das raízes. O comprimento e o diâmetro de raízes tuberosas foram avaliados em 10 raízes, sendo realizada a medida do comprimento de uma extremidade à outra, empregando-se uma régua graduada. $\mathrm{O}$ diâmetro médio das raízes foi obtido utilizando-se um paquímetro na porção mediana da raiz tuberosa. 
Tabela 1 - Análise granulométrica e química da camada arável $(0-20 \mathrm{~cm})$ do solo Argissolo Vermelho-Amarelo utilizado no experimento'

\begin{tabular}{|c|c|c|c|c|c|c|c|c|c|c|}
\hline \multicolumn{11}{|c|}{ Análise granulométrica $\left(\mathrm{dag}_{\mathrm{kg}}{ }^{-1}\right)$} \\
\hline \multicolumn{2}{|c|}{ Argila } & & Silte & & Areia fina & $\operatorname{Ar}$ & rrossa & \multicolumn{3}{|c|}{ Classificação textural } \\
\hline \multicolumn{2}{|c|}{34} & \multicolumn{2}{|r|}{12} & \multicolumn{2}{|r|}{18} & \multicolumn{2}{|c|}{36} & \multicolumn{3}{|c|}{ Franco-Argiloarenoso } \\
\hline \multicolumn{11}{|c|}{ Análise química } \\
\hline $\mathrm{pH}$ & $\mathrm{P}^{2} /$ & $\mathrm{K}^{+2} /$ & $\mathrm{H}+\mathrm{Al}^{\underline{3} /}$ & $\mathrm{Al}^{3+} \underline{4}$ & $\mathrm{Ca}^{2+4}$ & $\mathrm{Mg}^{2+} \underline{4}$ & $\mathrm{CTC}_{\text {total }}$ & $\mathrm{V}$ & $\mathrm{m}$ & $\mathrm{MO}$ \\
\hline$\left(\mathrm{H}_{2} \mathrm{O}\right)$ & \multicolumn{2}{|c|}{$\left(\mathrm{mg} \mathrm{dm}^{-3}\right)$} & \multicolumn{5}{|c|}{$\left(\mathrm{cmol}_{\mathrm{c}} \mathrm{dm}^{-3}\right)$} & \multicolumn{2}{|c|}{$(\%)$} & $\left(\right.$ dag kg $\left.{ }^{-1}\right)$ \\
\hline 5,5 & 7,4 & 76 & 2,7 & 0,0 & 2,8 & 0,8 & 3,61 & 56 & 0 & 3,68 \\
\hline
\end{tabular}

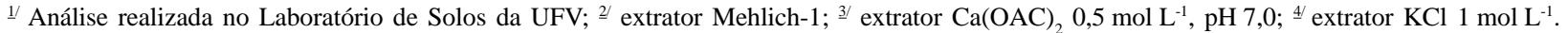

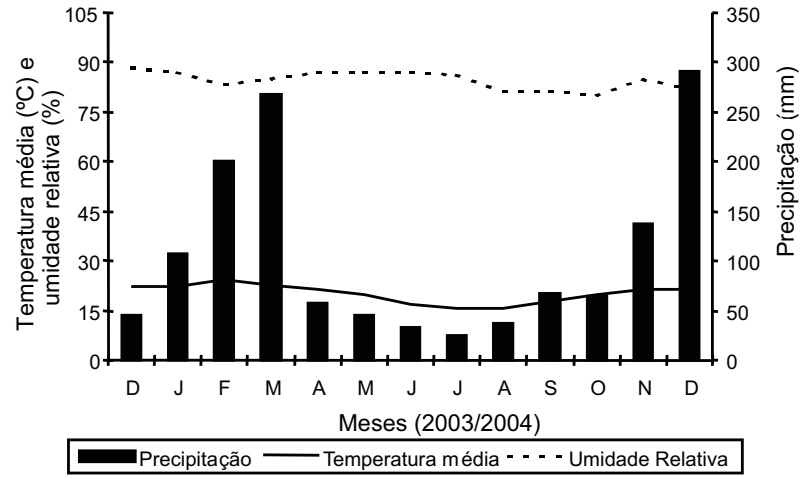

Figura 1 - Precipitação pluvial (mm), umidade relativa do ar (\%) e temperatura média $\left({ }^{\circ} \mathrm{C}\right)$ no período de condução do experimento (dezembro de 2003 a dezembro de 2004). Viçosa-MG. Dados obtidos da estação meteorológica pertencente ao Departamento de Engenharia Agrícola da UFV.

A análise estatística foi realizada separadamente para cada experimento, utilizandose o programa SAEG (Ribeiro Jr., 2004). As variáveis número de raízes, comprimento e diâmetro de raizes tuberosas foram submetidas à análise de variância $(\mathrm{p}<0,05)$. As médias dos tratamentos, quando significativas, foram comparadas pelo teste de Tukey a $5 \%$ de probabilidade. Quanto às características altura de planta e diâmetro de caule, adotou-se o esquema de parcelas subdivididas no tempo, sendo os tratamentos alocados nas parcelas, e as épocas de avaliação, nas subparcelas. A partir de valores estimados, foram elaboradas curvas de regressão com as características altura de plantas e diâmetro de caule em função do tempo.

\section{RESULTADOS E DISCUSSÃO}

Quanto ao número de comprimento de raízes por planta de mandioca, verificou-se que não houve diferença entre as médias quando a cultura foi mantida durante todo o ciclo livre da interferência (testemunha 1), ou quando ela ficou livre das plantas daninhas apenas até os 25 DAP (CPD 25 DAP), 50 DAP (CPD 50 DAP) ou 75 DAP (CPD 75 DAP) (Tabela 4). Esses resultados foram contrastantes com aqueles observados no segundo experimento (Tabela 5).

Comparando os valores da altura de plantas no primeiro experimento, verifica-se que não houve diferença entre os tratamentos quando se manteve a cultura livre de plantas daninhas até os 75 dias após o plantio (DAP). A partir dos 100 dias, de modo geral, os tratamentos que mantiveram a cultura durante todo o ciclo livre de plantas daninhas (testemunha 1) e em convivência de 25 dias após o plantio com essas plantas apresentaram as maiores alturas, mantendo-se até a época da colheita com 2,43 e 2,42 m, respectivamente (Tabela 6). Normalmente, a mandioca pode ser colhida em larga faixa de tempo, variando de 6 a 24 meses, dependendo de cultivar, região, tipos de uso, tratos dispensados e demanda de mercado (Albuquerque et al., 2009). Contudo, quando mantidos livres de plantas daninhas durante todo o ciclo (testemunha 1) ou em convivência com plantas daninhas por apenas 25 DAP, estes tratamentos apresentaram os maiores valores de diâmetro de caule, mantendo-se até a época da colheita com 2,67 e $2,55 \mathrm{~cm}$, respectivamente (Tabela 7).

No segundo experimento, de modo geral, os tratamentos livres de plantas daninhas durante todo o ciclo (testemunha 1) e também aqueles livres delas até 100 e 125 DAP apresentaram os maiores valores para altura de plantas (Tabela 8). Em experimentos realizados 
Tabela 2 - Descrição dos tratamentos avaliados no experimento 1. Viçosa-MG, 2006

\begin{tabular}{|c|c|}
\hline Tratamento & Descrição \\
\hline CPD 0 (Testemunha 1$)^{\frac{1}{}}$ & Cultura mantida sempre no limpo \\
\hline $\mathrm{CPD}^{3 /} 25^{4 /}$ & Convivência com plantas daninhas durante 25 dias após o plantio \\
\hline CPD 50 & Convivência com plantas daninhas durante 50 dias após o plantio \\
\hline CPD 75 & Convivência com plantas daninhas durante 75 dias após o plantio \\
\hline CPD 100 & Convivência com plantas daninhas durante 100 dias após o plantio \\
\hline CPD 125 & Convivência com plantas daninhas durante 125 dias após o plantio \\
\hline CPD350 DAP (testemunha 2) $\stackrel{2 /}{ }$ & Convivência com plantas daninhas durante todo o ciclo \\
\hline
\end{tabular}

1/ Cultivo livre de planta daninha durante todo o ciclo; $\stackrel{2}{2}$ cultivo com plantas daninhas durante todo o ciclo; ${ }^{2} /$ convivência com plantas daninhas; $\stackrel{4}{4}$ dias após o plantio.

Tabela 3 - Descrição dos tratamentos avaliados no experimento 2. Viçosa-MG, 2006

\begin{tabular}{|c|c|}
\hline Tratamento & Descrição \\
\hline LPD0 (testemunha 1) ${ }^{1 /}$ & Cultura mantida sempre no limpo até o final do ciclo \\
\hline Testemunha $2 \stackrel{2}{\prime}$ & Convivência com plantas daninhas durante todo o ciclo \\
\hline $\mathrm{LPD}^{3 /} 25^{4 /}$ & Livre de plantas daninhas 25 dias após o plantio \\
\hline LPD 50 & Livre de plantas daninhas 50 dias após o plantio \\
\hline LPD 75 & Livre de plantas daninhas 75 dias após o plantio \\
\hline LPD 100 & Livre de plantas daninhas 100 dias após o plantio \\
\hline LPD 125 & Livre de plantas daninhas 125 dias após o plantio \\
\hline
\end{tabular}

르 Cultivo livre de planta daninha durante todo o ciclo; $\stackrel{2}{2}$ cultivo com plantas daninhas durante todo o ciclo; $\underline{3}$ livre de plantas daninhas;

4/ dias após o plantio.

Tabela 4 - Médias de número de raízes por planta (NRP), comprimento de raiz (CR) e diâmetro de raiz (DR) do cultivar de mandioca Cacauzinha, referente ao experimento 1. Viçosa-MG, 2006

\begin{tabular}{|c|c|c|c|}
\hline Tratamento & NRP & $\mathrm{CR}(\mathrm{cm})$ & $\mathrm{DR}(\mathrm{cm})$ \\
\hline (Testemunha 1) ${ }^{1 /}$ & $3,90 \mathrm{~A}$ & $39,10 \mathrm{~A}$ & $3,47 \mathrm{~A}$ \\
\hline $\mathrm{CPD}^{3 /} 25^{4 /}$ & $3,72 \mathrm{~A}$ & $37,03 \mathrm{~A}$ & $3,51 \mathrm{~A}$ \\
\hline CPD 50 & $3,12 \mathrm{~A}$ & $32,70 \mathrm{~A}$ & $2,45 \mathrm{~B}$ \\
\hline CPD 75 & $2,92 \mathrm{AB}$ & $29,75 \mathrm{~A}$ & $2,35 \mathrm{~B}$ \\
\hline CPD 100 & $1,31 \mathrm{C}$ & $18,35 \mathrm{~B}$ & $2,41 \mathrm{~B}$ \\
\hline CPD 125 & $1,95 \mathrm{BC}$ & $19,10 \mathrm{~B}$ & $2,25 \mathrm{~B}$ \\
\hline CPD350DAP (testemunha 2) ${ }^{2 /}$ & $0 \mathrm{C}$ & $0 \mathrm{C}$ & $0 \mathrm{C}$ \\
\hline $\mathrm{CV}(\%)$ & 19,61 & 16,75 & 13,33 \\
\hline
\end{tabular}

Médias seguidas de mesma letra na coluna não diferem entre si a $5 \%$ de probabilidade pelo teste de Tukey. ${ }^{1 /}$ Cultivo livre de planta daninha durante todo o ciclo; $\stackrel{2 /}{ }$ cultivo com plantas daninhas durante todo o ciclo; $\stackrel{3 /}{ }$ convivência com plantas daninhas; ${ }^{4 /}$ dias após o plantio.

no Estado de Roraima, caracterizando 10 variedades de mandioca-mansa aos 13 meses, Albuquerque et al. (2009) verificaram que as alturas das plantas variaram de 1,33 a 2,62 m, e o diâmetro do caule, entre 3,70 e $5,07 \mathrm{~cm}$. Otsubo et al. (2007), ao trabalharem com sete cultivares de mandioca no município de
Dourados em 2003, observaram que os materiais que apresentaram as maiores alturas se destacaram na produção da parte aérea - essa observação demonstra correlação positiva entre esses fatores. Gomes et al. (2007) detectaram que a altura da planta está relacionada à produção de parte aérea e raiz. No segundo 
Tabela 5 - Médias de número de raízes por planta (NRP), comprimento de raiz (CR) e diâmetro de raiz (DR) do cultivar de mandioca Cacauzinha, referente ao experimento 2. Viçosa-MG, 2006

\begin{tabular}{|c|c|c|c|}
\hline Tratamento & NRP & $\mathrm{CR}(\mathrm{cm})$ & $\mathrm{DR}(\mathrm{cm})$ \\
\hline (Testemunha $1^{1}$ / & $3,85 \mathrm{AB}$ & $31,00 \mathrm{~A}$ & $2,95 \mathrm{~A}$ \\
\hline Testemunha $2^{2} /$ & $0 \mathrm{C}$ & $0 \mathrm{~B}$ & $0 \mathrm{~B}$ \\
\hline $\mathrm{LPD}^{3 /} 25^{4 /}$ & $2,65 \mathrm{~B}$ & $24,80 \mathrm{~A}$ & $2,65 \mathrm{~A}$ \\
\hline LPD 50 & $3,80 \mathrm{AB}$ & $32,35 \mathrm{~A}$ & $3,17 \mathrm{~A}$ \\
\hline LPD 125 & $3,90 \mathrm{AB}$ & $31,62 \mathrm{~A}$ & $2,72 \mathrm{~A}$ \\
\hline LPD 150 & $4,17 \mathrm{~A}$ & $25,00 \mathrm{~A}$ & $2,72 \mathrm{~A}$ \\
\hline LPD 125 & $3,72 \mathrm{AB}$ & $33,50 \mathrm{~A}$ & $3,12 \mathrm{~A}$ \\
\hline $\mathrm{CV}(\%)$ & 19,23 & 21,24 & 17,34 \\
\hline
\end{tabular}

Médias seguidas de mesma letra na coluna não diferem significativamente a $5 \%$ de probabilidade pelo teste de Tukey. ${ }^{1 /}$ Cultivo livre de planta daninha durante todo o ciclo; $\stackrel{2}{\prime}$ cultivo com plantas daninhas durante todo o ciclo; $\underline{3}^{3}$ convivência com plantas daninhas; 4/ dias após o plantio. experimento, para a característica diâmetro de caule, observou-se que durante todas as épocas de avaliação os tratamentos com capina durante todo o ciclo e aqueles livres de plantas daninhas por 100 e 125 dias depois do plantio apresentaram as maiores médias (Tabela 9). Já nos demais tratamentos, os dados referentes a diâmetros de caule foram menores. Isso demonstrou que a maior convivência das plantas daninhas com a mandioca reduz o crescimento de diâmetro do caule.

Nas Figuras 2, 3, 4 e 5, observa-se, em todos os tratamentos de ambos os experimentos, que as características altura de planta e diâmetro de caule seguem o modelo cúbico quadrático. Esse modelo mostra que na fase inicial de desenvolvimento da cultura houve aumento em altura de planta e diâmetro de caule,

Tabela 6 - Médias de altura (m) de plantas de mandioca cultivar Cacauzinha, com avaliações realizadas a intervalos de 25 dias desde o plantio até a colheita, no experimento 1. Viçosa-MG, 2006

\begin{tabular}{|c|c|c|c|c|c|c|c|c|c|c|c|c|}
\hline \multirow{2}{*}{ Tratamento } & \multicolumn{12}{|c|}{ Dias após o plantio } \\
\hline & 75 & 100 & 125 & 150 & 175 & 200 & 225 & 250 & 275 & 300 & 325 & 350 \\
\hline CPD 0 (Test. 1) $)^{\frac{1}{}}$ & $0,56 \mathrm{~A}$ & $0,98 \mathrm{~A}$ & $1,30 \mathrm{~A}$ & $1,41 \mathrm{~A}$ & $1,47 \mathrm{~A}$ & $1,55 \mathrm{~A}$ & $1,57 \mathrm{~A}$ & $1,62 \mathrm{~A}$ & $1,83 \mathrm{~A}$ & $2,00 \mathrm{~A}$ & $2,20 \mathrm{~A}$ & $2,43 \mathrm{~A}$ \\
\hline $\mathrm{CPD}^{3 /} 25^{4 /}$ & $0,47 \mathrm{~A}$ & $0,92 \mathrm{~A}$ & $1,25 \mathrm{~A}$ & $1,31 \mathrm{~A}$ & $1,39 \mathrm{~A}$ & $1,45 \mathrm{~A}$ & $1,48 \mathrm{~A}$ & $1,54 \mathrm{~A}$ & $1,56 \mathrm{~B}$ & $1,73 \mathrm{~B}$ & $2,10 \mathrm{~A}$ & $2,42 \mathrm{~A}$ \\
\hline CPD 50 & $0,49 \mathrm{~A}$ & $0,77 \mathrm{~B}$ & $0,96 \mathrm{~B}$ & $1,01 \mathrm{~B}$ & $1,08 \mathrm{~B}$ & $1,16 \mathrm{~B}$ & $1,20 \mathrm{~B}$ & $1,24 \mathrm{~B}$ & $1,31 \mathrm{C}$ & $1,39 \mathrm{C}$ & $1,60 \mathrm{C}$ & $2,09 \mathrm{BC}$ \\
\hline CPD 75 & $0,48 \mathrm{~A}$ & $0,70 \mathrm{~B}$ & $0,82 \mathrm{BC}$ & $0,86 \mathrm{CD}$ & $0,93 \mathrm{C}$ & $0,99 \mathrm{C}$ & $1,03 \mathrm{C}$ & $1,06 \mathrm{C}$ & $1,10 \mathrm{D}$ & $1,26 \mathrm{C}$ & $1,73 \mathrm{BC}$ & $1,97 \mathrm{C}$ \\
\hline CPD 100 & $0,56 \mathrm{~A}$ & $0,69 \mathrm{BC}$ & $0,90 \mathrm{~B}$ & $0,95 \mathrm{BC}$ & $1,00 \mathrm{BC}$ & $1,08 \mathrm{BC}$ & $1,15 \mathrm{BC}$ & $1,17 \mathrm{BC}$ & $1,22 \mathrm{CD}$ & $1,39 \mathrm{C}$ & $1,79 \mathrm{~B}$ & $2,18 \mathrm{~B}$ \\
\hline CPD 125 & $0,51 \mathrm{~A}$ & $0,62 \mathrm{C}$ & $0,73 \mathrm{CD}$ & $0,73 \mathrm{DE}$ & $0,78 \mathrm{D}$ & $0,81 \mathrm{D}$ & $0,85 \mathrm{D}$ & $0,89 \mathrm{D}$ & $0,93 \mathrm{E}$ & $1,06 \mathrm{D}$ & $1,22 \mathrm{D}$ & $1,55 \mathrm{D}$ \\
\hline CPD350DAP(Test. 2) $)^{\frac{2}{1}}$ & $0,53 \mathrm{~A}$ & $0,57 \mathrm{C}$ & $0,61 \mathrm{D}$ & $0,64 \mathrm{E}$ & $0,64 \mathrm{D}$ & $0,65 \mathrm{E}$ & $0,67 \mathrm{E}$ & $0,68 \mathrm{E}$ & $0,74 \mathrm{~F}$ & $0,86 \mathrm{E}$ & $1,12 \mathrm{D}$ & $1,47 \mathrm{D}$ \\
\hline
\end{tabular}

Médias seguidas de mesma letra na coluna não diferem significativamente a $5 \%$ de probabilidade pelo teste de Tukey. ${ }^{1 /}$ Cultivo livre de planta daninha durante todo o ciclo; ${ }^{2 /}$ cultivo com plantas daninhas durante todo o ciclo; ${ }^{3 /}$ convivência com plantas daninhas; ${ }^{4 /}$ dias após o plantio.

Tabela 7 - Médias de diâmetro de caule $(\mathrm{cm})$ de plantas de mandioca cultivar Cacauzinha, com avaliações realizadas a intervalos de 25 dias desde o plantio até a colheita, no experimento 1. Viçosa-MG, 2006

\begin{tabular}{|c|c|c|c|c|c|c|c|c|c|c|c|c|}
\hline \multirow{2}{*}{ Tratamento } & \multicolumn{12}{|c|}{ Dias após o plantio } \\
\hline & 75 & 100 & 125 & 150 & 175 & 200 & 225 & 250 & 275 & 300 & 325 & 350 \\
\hline CPD 0 (Test. 1) $\frac{1}{1 /}$ & $1,21 \mathrm{~A}$ & $1,66 \mathrm{~A}$ & $1,79 \mathrm{~A}$ & $1,86 \mathrm{~A}$ & $1,91 \mathrm{~A}$ & $1,98 \mathrm{~A}$ & $2,00 \mathrm{~A}$ & $2,03 \mathrm{~A}$ & $2,08 \mathrm{~A}$ & $2,19 \mathrm{~A}$ & $2,43 \mathrm{~A}$ & $\overline{2,67 \mathrm{~A}}$ \\
\hline $\mathrm{CPD}^{3 /} 25^{4 /}$ & $1,12 \mathrm{~A}$ & $1,53 \mathrm{~A}$ & $1,73 \mathrm{~A}$ & $1,79 \mathrm{~A}$ & $1,85 \mathrm{~A}$ & $1,88 \mathrm{~A}$ & $1,91 \mathrm{~A}$ & $1,95 \mathrm{~A}$ & $1,98 \mathrm{~A}$ & $2,05 \mathrm{~A}$ & $2,25 \mathrm{~B}$ & $2,55 \mathrm{~A}$ \\
\hline CPD 50 & $0,69 \mathrm{~B}$ & $1,05 \mathrm{~B}$ & $1,33 \mathrm{~B}$ & $1,36 \mathrm{~B}$ & $1,43 \mathrm{~B}$ & $1,50 \mathrm{~B}$ & $1,55 \mathrm{~B}$ & $1,58 \mathrm{~B}$ & $1,61 \mathrm{~B}$ & $1,70 \mathrm{~B}$ & $2,16 \mathrm{~B}$ & $2,52 \mathrm{~A}$ \\
\hline CPD 75 & $0,56 \mathrm{BC}$ & $1,21 \mathrm{~B}$ & $1,28 \mathrm{~B}$ & $1,34 \mathrm{~B}$ & $1,35 \mathrm{~B}$ & $1,38 \mathrm{BC}$ & $1,40 \mathrm{BC}$ & $1,42 \mathrm{BC}$ & $1,44 \mathrm{C}$ & $1,49 \mathrm{C}$ & $1,67 \mathrm{C}$ & $1,98 \mathrm{~B}$ \\
\hline CPD 100 & $0,61 \mathrm{BC}$ & $0,82 \mathrm{C}$ & $0,97 \mathrm{C}$ & $1,04 \mathrm{C}$ & $1,17 \mathrm{C}$ & $1,25 \mathrm{C}$ & $1,33 \mathrm{C}$ & $1,35 \mathrm{C}$ & $1,37 \mathrm{C}$ & $1,49 \mathrm{C}$ & $1,70 \mathrm{C}$ & $2,05 \mathrm{~B}$ \\
\hline CPD 125 & $0,55 \mathrm{BC}$ & $0,63 \mathrm{D}$ & $0,67 \mathrm{D}$ & $0,70 \mathrm{D}$ & $0,72 \mathrm{D}$ & $0,76 \mathrm{D}$ & $0,79 \mathrm{D}$ & $0,81 \mathrm{D}$ & $0,85 \mathrm{D}$ & $0,93 \mathrm{D}$ & $1,04 \mathrm{D}$ & $1,34 \mathrm{C}$ \\
\hline CPD350DAP(Test. 2) 2! $^{\frac{2}{2}}$ & $0,51 \mathrm{C}$ & $0,56 \mathrm{D}$ & $0,58 \mathrm{D}$ & $0,60 \mathrm{D}$ & $0,61 \mathrm{D}$ & $0,63 \mathrm{D}$ & $0,64 \mathrm{D}$ & $0,65 \mathrm{D}$ & $0,68 \mathrm{E}$ & $0,77 \mathrm{D}$ & $1,07 \mathrm{D}$ & $1,41 \mathrm{C}$ \\
\hline
\end{tabular}

Médias seguidas de mesma letra na coluna não diferem significativamente a $5 \%$ de probabilidade pelo teste de Tukey. ${ }^{1 /}$ Cultivo livre de


o plantio. 
Tabela 8 - Médias de altura (m) de plantas de mandioca cultivar Cacauzinha, com avaliações realizadas a intervalos de 25 dias após o plantio, no experimento 2. Viçosa-MG, 2006

\begin{tabular}{|c|c|c|c|c|c|c|c|c|c|c|c|c|}
\hline \multirow{2}{*}{ Tratamento } & \multicolumn{12}{|c|}{ Dias após o plantio } \\
\hline & 75 & 100 & 125 & 150 & 175 & 200 & 225 & 250 & 275 & 300 & 325 & 350 \\
\hline Testemunha $1 \frac{1 /}{}$ & $1,21 \mathrm{~A}$ & $1,66 \mathrm{~A}$ & $1,79 \mathrm{~A}$ & $1,86 \mathrm{~A}$ & $1,91 \mathrm{~A}$ & $1,98 \mathrm{~A}$ & $2,00 \mathrm{~A}$ & $2,03 \mathrm{~A}$ & $2,08 \mathrm{~A}$ & $2,19 \mathrm{~A}$ & $2,43 \mathrm{~A}$ & $2,67 \mathrm{~A}$ \\
\hline Testemunha $2^{2} \stackrel{2}{ }$ & $1,12 \mathrm{~A}$ & $1,53 \mathrm{~A}$ & $1,73 \mathrm{~A}$ & $1,79 \mathrm{~A}$ & $1,85 \mathrm{~A}$ & $1,88 \mathrm{~A}$ & $1,91 \mathrm{~A}$ & $1,95 \mathrm{~A}$ & $1,98 \mathrm{~A}$ & $2,05 \mathrm{~A}$ & $2,25 \mathrm{~B}$ & $2,55 \mathrm{~A}$ \\
\hline $\mathrm{LPD}^{3 / /} 25^{4 /}$ & $0,69 \mathrm{~B}$ & $1,05 \mathrm{~B}$ & $1,33 \mathrm{~B}$ & $1,36 \mathrm{~B}$ & $1,43 \mathrm{~B}$ & $1,50 \mathrm{~B}$ & $1,55 \mathrm{~B}$ & $1,58 \mathrm{~B}$ & $1,61 \mathrm{~B}$ & $1,70 \mathrm{~B}$ & $2,16 \mathrm{~B}$ & $2,52 \mathrm{~A}$ \\
\hline LPD 50 & $0,56 \mathrm{BC}$ & $1,21 \mathrm{~B}$ & $1,28 \mathrm{~B}$ & $1,34 \mathrm{~B}$ & $1,35 \mathrm{~B}$ & $1,38 \mathrm{BC}$ & $1,40 \mathrm{BC}$ & $1,42 \mathrm{BC}$ & $1,44 \mathrm{C}$ & $1,49 \mathrm{C}$ & $1,67 \mathrm{C}$ & $1,98 \mathrm{~B}$ \\
\hline LPD 75 & $0,61 \mathrm{BC}$ & $0,82 \mathrm{C}$ & $0,97 \mathrm{C}$ & $1,04 \mathrm{C}$ & $1,17 \mathrm{C}$ & $1,25 \mathrm{C}$ & $1,33 \mathrm{C}$ & $1,35 \mathrm{C}$ & $1,37 \mathrm{C}$ & $1,49 \mathrm{C}$ & $1,70 \mathrm{C}$ & $2,05 \mathrm{~B}$ \\
\hline LPD 100 & $0,55 \mathrm{BC}$ & $0,63 \mathrm{D}$ & $0,67 \mathrm{D}$ & $0,70 \mathrm{D}$ & $0,72 \mathrm{D}$ & $0,76 \mathrm{D}$ & $0,79 \mathrm{D}$ & $0,81 \mathrm{D}$ & $0,85 \mathrm{D}$ & $0,93 \mathrm{D}$ & $1,04 \mathrm{D}$ & $1,34 \mathrm{C}$ \\
\hline LPD 125 & $0,51 \mathrm{C}$ & $0,56 \mathrm{D}$ & $0,58 \mathrm{D}$ & $0,60 \mathrm{D}$ & $0,61 \mathrm{D}$ & $0,63 \mathrm{D}$ & $0,64 \mathrm{D}$ & $0,65 \mathrm{D}$ & $0,68 \mathrm{E}$ & $0,77 \mathrm{D}$ & $1,07 \mathrm{D}$ & $1,41 \mathrm{C}$ \\
\hline
\end{tabular}

Médias seguidas de mesma letra na coluna não diferem significativamente a $5 \%$ de probabilidade pelo teste de Tukey. . ${ }^{1 /}$ Cultivo livre de planta daninha durante todo o ciclo; ${ }^{2 /}$ cultivo com plantas daninhas durante todo o ciclo; ${ }^{3 /}$ livre de plantas daninhas; ${ }^{4 /}$ dias após o plantio.

Tabela 9 - Médias de diâmetro (cm) de caule de plantas de mandioca cultivar Cacauzinha, com avaliações realizadas a intervalos de 25 dias após o plantio, no experimento 2. Viçosa-MG, 2006

\begin{tabular}{|c|c|c|c|c|c|c|c|c|c|c|c|c|}
\hline \multirow{2}{*}{ Tratamento } & \multicolumn{12}{|c|}{ Dias após o plantio } \\
\hline & 75 & 100 & 125 & 150 & 175 & 200 & 225 & 250 & 275 & 300 & 325 & 350 \\
\hline Testemunha $1^{\underline{1} /}$ & $1,36 \mathrm{~A}$ & $1,46 \mathrm{~A}$ & $1,50 \mathrm{~A}$ & $1,52 \mathrm{~A}$ & $1,53 \mathrm{~A}$ & $1,55 \mathrm{~A}$ & $1,56 \mathrm{~A}$ & $1,57 \mathrm{~A}$ & $1,69 \mathrm{~A}$ & $1,98 \mathrm{~A}$ & $2,30 \mathrm{~A}$ & $2,77 \mathrm{~A}$ \\
\hline Testemunha $2^{2} /$ & $0,50 \mathrm{BC}$ & $0,53 \mathrm{BC}$ & $0,56 \mathrm{BC}$ & $0,58 \mathrm{~B}$ & $0,60 \mathrm{~B}$ & $0,62 \mathrm{~B}$ & $0,63 \mathrm{~B}$ & $0,64 \mathrm{~B}$ & $0,68 \mathrm{~B}$ & $0,73 \mathrm{C}$ & $0,92 \mathrm{C}$ & $1,17 \mathrm{C}$ \\
\hline $\mathrm{LPD}^{3 /} 25^{4 /}$ & $0,44 \mathrm{C}$ & $0,48 \mathrm{C}$ & $0,50 \mathrm{C}$ & $0,53 \mathrm{~B}$ & $0,56 \mathrm{~B}$ & $0,58 \mathrm{~B}$ & $0,59 \mathrm{~B}$ & $0,62 \mathrm{~B}$ & $0,66 \mathrm{~B}$ & $0,74 \mathrm{C}$ & $0,91 \mathrm{C}$ & $1,16 \mathrm{C}$ \\
\hline LPD 50 & $0,61 \mathrm{~B}$ & $0,63 \mathrm{~B}$ & $0,64 \mathrm{~B}$ & $0,66 \mathrm{~B}$ & $0,69 \mathrm{~B}$ & $0,69 \mathrm{~B}$ & $0,71 \mathrm{~B}$ & $0,73 \mathrm{~B}$ & $0,80 \mathrm{~B}$ & $0,98 \mathrm{~B}$ & $1,17 \mathrm{~B}$ & $1,59 \mathrm{~B}$ \\
\hline LPD 75 & $0,61 \mathrm{~B}$ & $0,63 \mathrm{~B}$ & $0,64 \mathrm{~B}$ & $0,65 \mathrm{~B}$ & $0,68 \mathrm{~B}$ & $0,68 \mathrm{~B}$ & $0,69 \mathrm{~B}$ & $0,71 \mathrm{~B}$ & $0,79 \mathrm{~B}$ & $0,99 \mathrm{~B}$ & $1,19 \mathrm{~B}$ & $1,65 \mathrm{~B}$ \\
\hline LPD 100 & $1,34 \mathrm{~A}$ & $1,44 \mathrm{~A}$ & $1,51 \mathrm{~A}$ & $1,54 \mathrm{~A}$ & $1,55 \mathrm{~A}$ & $1,57 \mathrm{~A}$ & $1,59 \mathrm{~A}$ & $1,59 \mathrm{~A}$ & $1,68 \mathrm{~A}$ & $1,89 \mathrm{~A}$ & $2,30 \mathrm{~A}$ & $2,72 \mathrm{~A}$ \\
\hline LPD 125 & $1,35 \mathrm{~A}$ & $1,43 \mathrm{~A}$ & $1,49 \mathrm{~A}$ & $1,57 \mathrm{~A}$ & $1,59 \mathrm{~A}$ & $1,60 \mathrm{~A}$ & $1,61 \mathrm{~A}$ & $1,62 \mathrm{~A}$ & $1,70 \mathrm{~A}$ & $1,93 \mathrm{~A}$ & $2,33 \mathrm{~A}$ & $2,70 \mathrm{~A}$ \\
\hline
\end{tabular}

Médias seguidas de mesma letra na coluna não diferem significativamente a $5 \%$ de probabilidade pelo teste de Tukey. ${ }^{1 /}$ Cultivo livre de planta daninha durante todo o ciclo; $\stackrel{2 /}{ }$ cultivo com plantas daninhas durante todo o ciclo; $\stackrel{3}{2}$ livre de plantas daninhas; ${ }^{4 /}$ dias após o plantio.

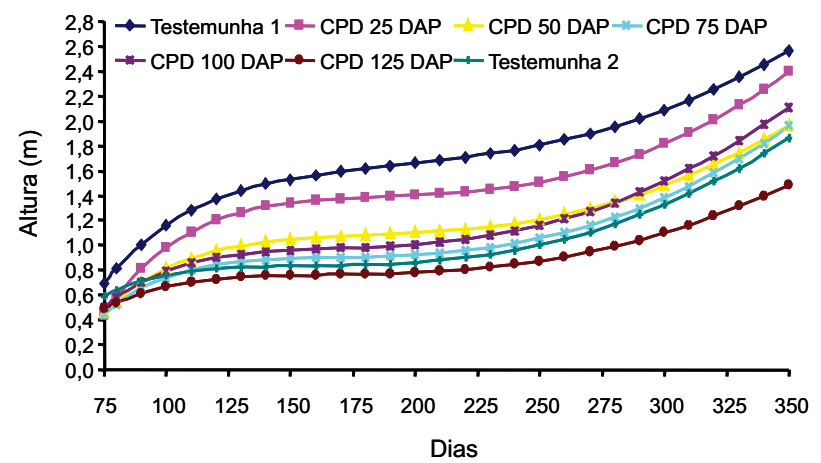

Figura 2 - Valores estimados para altura de plantas de mandioca em função do tempo (em dias) após o plantio, para diferentes condições de cultivo: livre de plantas daninhas durante todo o ciclo (testemunha 1), convivência durante todo o ciclo com plantas daninhas (testemunha 2) e culturas em convivência com plantas daninhas (CPD) por 25, 50, 75, 100, 125 e 150 dias após o plantio (DAP), para o experimento 1. Modelo cúbico-raiz ( $\hat{\mathrm{Y}}=\mathrm{a}+\mathrm{bx}^{0,5}+\mathrm{cx}+$ $\mathrm{dx}^{1,5}$ ). Viçosa-MG, 2006.

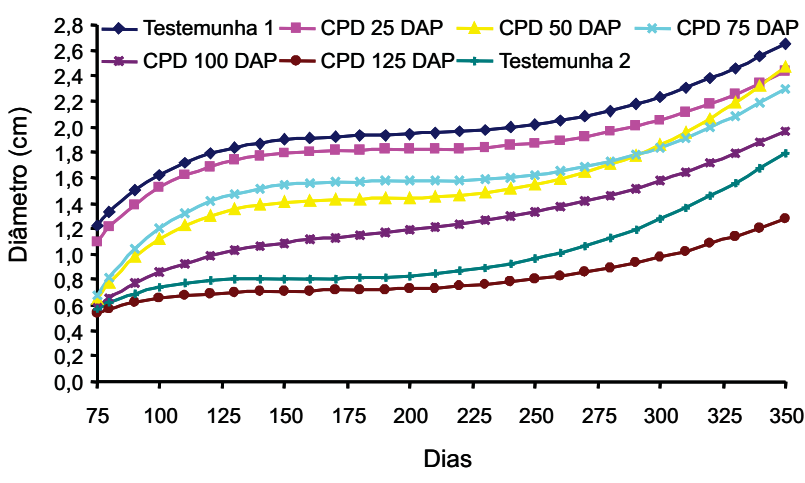

Figura 3 - Valores estimados para diâmetro de caule de plantas de mandioca em função do tempo (em dias) após o plantio, para diferentes condições de cultivo: livre de plantas daninhas durante todo o ciclo (testemunha 1), convivência durante todo o ciclo com plantas daninhas (testemunha 2) e culturas em convivência com plantas daninhas (CPD) por 25, 50, 75, 100, 125 e 150 dias após o plantio (DAP), para o experimento 2. Modelo cúbico-raiz ( $\hat{\mathrm{Y}}=\mathrm{a}+\mathrm{bx}^{0,5}+\mathrm{cx}+$ $\left.\mathrm{dx}^{1,5}\right)$. Viçosa-MG, 2006. 




Figura 4 - Valores estimados para altura de plantas de mandioca em função do tempo (em dias) após o plantio, para diferentes condições de cultivo: livre de plantas daninhas durante todo o ciclo (testemunha 1), convivência durante todo o ciclo com plantas daninhas (testemunha 2) e culturas livres de plantas daninhas (LPD) por 25, 50, 75, 100, 125 e 150 dias após o plantio (DAP), para o experimento 2. Modelo cúbico$\operatorname{raiz}\left(\hat{\mathrm{Y}}=\mathrm{a}+\mathrm{bx}^{0,5}+\mathrm{cX}+\mathrm{dx}^{1,5}\right)$. Viçosa-MG, 2006.

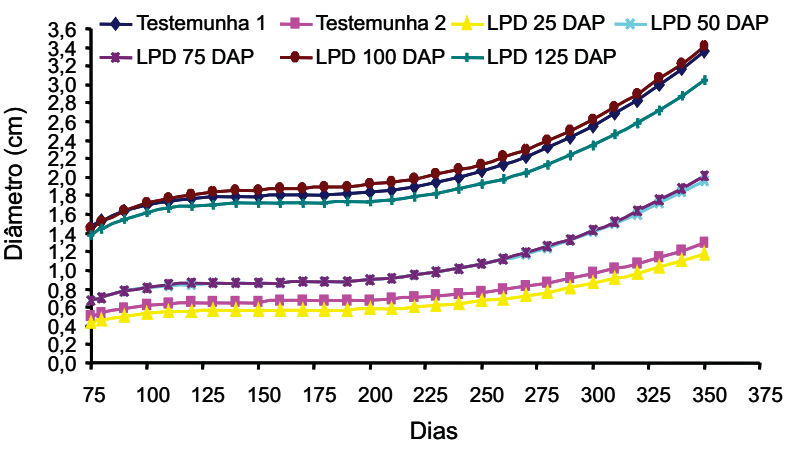

Figura 5 - Valores estimados para diâmetro de caule de plantas de mandioca em função do tempo (em dias) após o plantio, para diferentes condições de cultivo: livre de plantas daninhas durante todo o ciclo (testemunha 1), convivência durante todo o ciclo com plantas daninhas (testemunha 2) e culturas livres de plantas daninhas (LPD) por 25, 50, 75, 100, 125 e 150 dias após o plantio (DAP), para o experimento 2. Modelo cúbico-raiz ( $\left.\hat{\mathrm{Y}}=\mathrm{a}+\mathrm{bx}^{0,5}+\mathrm{cx}+\mathrm{dx}^{1,5}\right)$. Viçosa-MG, 2006.

Tabela 10 - Equações de regressão relacionando altura de plantas e diâmetro de caule em função do tempo (em dias), para o experimento 1 - Modelo cúbico-raiz ( $\left.\hat{\mathrm{Y}}=\mathrm{a}+\mathrm{bx}^{0,5}+\mathrm{cx}+\mathrm{dx}^{1,5}\right)$

\begin{tabular}{|c|c|c|c|}
\hline Tratamento & Variável & Equação de Regressão & $\mathrm{R}^{2}$ \\
\hline \multirow{2}{*}{1} & Altura & $\hat{\mathrm{Y}}=-12,23881+2,922371 * * \mathrm{x}^{0,5}-0,209163 * * \mathrm{x}+0,005092 * * \mathrm{x}^{1,5}$ & 0,96 \\
\hline & Diâmetro & $\hat{\mathrm{Y}}=-11,0926+2,8248^{* *} \mathrm{x}^{0,5}-0,205367 * * \mathrm{x}+0,005006^{* *} \mathrm{x}^{1,5}$ & 0,79 \\
\hline \multirow{2}{*}{2} & Altura & $\hat{\mathrm{Y}}=-16,1957+3,8242 * * \mathrm{x}^{0,5}-0,27878 * * \mathrm{x}+0,006814 * * \mathrm{x}^{1,5}$ & 0,92 \\
\hline & Diâmetro & $\hat{\mathrm{Y}}=-11,6407+2,9128 * * \mathrm{x}^{0,5}-0,210459 * * \mathrm{x}+0,005077 * * \mathrm{x}^{1,5}$ & 0,86 \\
\hline \multirow{2}{*}{3} & Altura & $\hat{\mathrm{Y}}=-11,1428+2,6993 * * \mathrm{x}^{0,5}-0,200338 * * \mathrm{x}+0,0049987 * * \mathrm{x}^{1,5}$ & 0,95 \\
\hline & Diâmetro & $\hat{\mathrm{Y}}=-15,0603+3,66025 * * \mathrm{x}^{0,5}-0,271539 * * \mathrm{x}+0,0067327 * * \mathrm{x}^{1,5}$ & 0,94 \\
\hline \multirow{2}{*}{4} & Altura & $\hat{\mathrm{Y}}=-10,5459+2,63336^{* *} \mathrm{x}^{0,5}-0,202648^{* *} \mathrm{x}+0,00521728^{* *} \mathrm{x}^{1,5}$ & 0,91 \\
\hline & Diâmetro & $\hat{\mathrm{Y}}=-15,2449+3,63558^{* *} \mathrm{x}^{0,5}-0,262098^{* *} \mathrm{x}+0,00630146^{* *} \mathrm{x}^{1,5}$ & 0,86 \\
\hline \multirow{2}{*}{5} & Altura & $\hat{\mathrm{Y}}=-10,4296+2,61838^{* *} \mathrm{x}^{0,5}-0,201765^{* *} \mathrm{x}+0,00521930^{* *} \mathrm{x}^{1,5}$ & 0,93 \\
\hline & Diâmetro & $\hat{\mathrm{Y}}=-7,2948+1,80433 * * x^{0,5}-0,132097 * * x+0,0033193 * * x^{1,5}$ & 0,88 \\
\hline \multirow{2}{*}{6} & Altura & $\hat{\mathrm{Y}}=-6,50715+1,6842 * * \mathrm{x}^{0,5}-0,13056^{* *} \mathrm{x}+0,00338779 * * \mathrm{x}^{1,5}$ & 0,93 \\
\hline & Diâmetro & $\hat{\mathrm{Y}}=-4,3585+1,1893 * * \mathrm{x}^{0,5}-0,093327 * * \mathrm{x}+0,0040967 * * \mathrm{x}^{1,5}$ & 0,81 \\
\hline \multirow{2}{*}{7} & Altura & $\hat{\mathrm{Y}}=-7,17013+1,91563 * * \mathrm{x}^{0,5}-0,153231 * * \mathrm{x}+0,0040967 * * \mathrm{x}^{1,5}$ & 0,89 \\
\hline & Diâmetro & $\hat{\mathrm{Y}}=-7,09147+1,8926^{* *} \mathrm{x}^{0,5}-0,151334 * * \mathrm{x}+0,00403815^{* *} \mathrm{x}^{1,5}$ & 0,87 \\
\hline
\end{tabular}

** Significativo a $1 \%$ de probabilidade pelo teste $\mathrm{t}$.

seguido por um período de reduzido incremento no crescimento da planta. Após esse período, a altura de planta e o diâmetro de caule retornaram ao seu crescimento. Portanto, acreditase que durante o intervalo intermediário de desenvolvimento da cultura (125 a 250 DAP) as plantas de mandioca encontravam-se em repouso vegetativo. De acordo com Peixoto (1999), essa é uma das fases de desenvolvimento da mandioca em que a emissão foliar diminui e a queda de folhas por senescência aumenta, reduzindo a área foliar total. Essa característica é mais pronunciada em regiões que apresentam oscilações significativas de 
Tabela 11 - Equações de regressão de diâmetro de caule e altura de plantas de mandioca em função do tempo (em dias), para o experimento 2 - Modelo cúbico-raiz ( $\left.\hat{\mathrm{Y}}=\mathrm{a}+\mathrm{bx}^{0,5}+\mathrm{cx}+\mathrm{dx} \mathrm{x}^{1,5}\right)$

\begin{tabular}{|c|c|c|c|}
\hline Tratamento & Variável & Equação de Regressão & $\mathrm{R}^{2}$ \\
\hline \multirow{2}{*}{1} & Altura & $\hat{\mathrm{Y}}=-2,4886+3,36081 * * \mathrm{x}^{0,5}-0,277482 * * \mathrm{x}+0,00767787 * * \mathrm{x}^{1,5}$ & 0,96 \\
\hline & Diâmetro & $\hat{\mathrm{Y}}=-9,81924+2,79759^{*} * \mathrm{x}^{0,5}-0,225038^{* *} \mathrm{x}+0,00604779^{*} \mathrm{x}^{1,5}$ & 0,79 \\
\hline \multirow{2}{*}{2} & Altura & $\hat{\mathrm{Y}}=-5,36836+1,48136^{* *} \mathrm{x}^{0,5}-0,120495^{* *} \mathrm{x}+0,0032729 * * \mathrm{x}^{1,5}$ & 0,92 \\
\hline & Diâmetro & $\hat{\mathrm{Y}}=-4,4803+1,22792 * * x^{0,5}-0,0978517 * * x+0,00260286^{* *} x^{1,5}$ & 0,86 \\
\hline \multirow{2}{*}{3} & Altura & $\hat{\mathrm{Y}}=-8,82793+2,3263^{* *} \mathrm{x}^{0,5}-0,18613^{* *} \mathrm{x}+0,00499314 * * \mathrm{x}^{1,5}$ & 0,95 \\
\hline & Diâmetro & $\hat{\mathrm{Y}}=-4,17750+1,14155^{* *} \mathrm{x}^{0,5}-0,091661 * * \mathrm{x}+0,00245577^{* *} \mathrm{x}^{1,5}$ & 0,94 \\
\hline \multirow{2}{*}{4} & Altura & $\hat{\mathrm{Y}}=-9,23246+2,44585^{* *} \mathrm{x}^{0,5}-0,19800 * * \mathrm{x}+0,0053479^{*} \mathrm{x}^{1,5}$ & 0,91 \\
\hline & Diâmetro & $\hat{\mathrm{Y}}=-6,44042+1,78048^{* *} \mathrm{x}^{0,5}-0,145051^{* *} \mathrm{x}+0,00394995^{* *} \mathrm{x}^{1,5}$ & 0,86 \\
\hline \multirow{2}{*}{5} & Altura & $\hat{\mathrm{Y}}=-8,36674+2,23575^{* *} \mathrm{x}^{0,5}-0,182361^{* *} \mathrm{x}+0,00489823^{* *} \mathrm{x}^{1,5}$ & 0,93 \\
\hline & Diâmetro & $\hat{\mathrm{Y}}=-7,0352+1,93057 * * \mathrm{x}^{0,5}-0,15725 * * \mathrm{x}+0,00427144 * * \mathrm{x}^{1,5}$ & 0,88 \\
\hline \multirow{2}{*}{6} & Altura & $\hat{\mathrm{Y}}=-13,3613+3,4486 * * \mathrm{x}^{0,5}-0,275072 * * \mathrm{x}+0,00731366^{* *} \mathrm{x}^{1,5}$ & 0,93 \\
\hline & Diâmetro & $\hat{\mathrm{Y}}=-10,3423+2,88775^{* *} \mathrm{x}^{0,5}-0,229011^{* *} \mathrm{x}+0,00609119^{* *} \mathrm{x}^{1,5}$ & 0,81 \\
\hline \multirow{2}{*}{7} & Altura & $\hat{\mathrm{Y}}=-12,0359+3,11432 * * \mathrm{x}^{0,5}-0,248265 * * \mathrm{x}+0,00661017 * * \mathrm{x}^{1,5}$ & 0,89 \\
\hline & Diâmetro & $\hat{\mathrm{Y}}=-9,42636+2,65671^{*} \mathrm{x}^{0,5}-0,211346 * * \mathrm{x}+0,00561077^{*} * \mathrm{x}^{1,5}$ & 0,87 \\
\hline
\end{tabular}

** Significativo a $1 \%$ de probabilidade pelo teste $\mathrm{t}$.

temperatura e/ou deficiência hídrica durante o ano. Na Figura 1, o intervalo situou-se entre os meses de maio e outubro de 2004, período em que a temperatura e precipitação pluvial apresentaram os menores índices.

Nas Tabelas 10 e 11 são apresentadas as equações referentes aos valores para altura e diâmetro de caule das plantas de mandioca em função dos períodos de convivência com as plantas daninhas.

Não existem relatos sobre qual seria o tamanho ideal das plantas de mandioca. Plantas mais altas podem favorecer a realização de alguns tratos culturais e a colheita; contudo, também são mais suscetiveis ao acamamento, o que dificulta o processo de colheita (Gomes et al., 2007).

Segundo Peixoto et al. (2005), há correlação positiva entre parte aérea fotossinteticamente ativa e acúmulo de reservas nas raízes.

Diante dos resultados, conclui-se que é necessário manter a cultura da mandioca livre das plantas daninhas pelo menos até os 75 DAP, a fim de permitir o desenvolvimento normal das raízes; que o controle das plantas daninhas nessa cultura deve ser iniciado aos
25 dias após o plantio; e que a convivência das plantas daninhas com a cultura da mandioca por intervalo igual ou superior a 50 DAP afeta de forma negativa a altura das plantas, o diâmetro de caule e o número e comprimento das raízes de plantas de mandioca.

\section{LITERATURA CITADA}

ALBUQUERQUE, J. A. A. et al. Caracterização morfológica e agronômica de clones de mandioca cultivados no Estado de Roraima. R. Bras. Ci. Agr., v. 4, n. 4, p. 388-394, 2009.

ALBURQUERQUE, J. A. A. et al. Interferência de plantas daninhas sobre a produtividade da mandioca (Manihot esculenta). Planta Daninha, v. 26, n. 2, p. 279-289, 2008.

AZEVÊDO, C. L. L. et al. Levantamento de plantas daninhas na cultura da mandioca, em um ecossistema semi-árido do Estado da Bahia. Magistra, v. 12, n. 1/2, P. 41-49, 2000.

\section{COMISSÃO DE FERTILIDADE DO SOLO DO ESTADO} DE MINAS GERAIS - CFSEMG. Recomendações para uso de corretivos e fertilizantes em Minas Gerais: $5^{\text {a }}$ aproximação. Viçosa, MG: 1999. 360 p.

FOOD AND AGRICULTURE ORGANIZATION OF THE UNITED NATIONS - FAO. Cassava for food and energy security. Disponível em: <http://www.fao.org/newsroom/en/ news/2009/1000899/index.html>. Acesso em: 05 maio 2011. 
GOMES, C. N. et al. Caracterização morfoagronômica e coeficientes de trilha de caracteres componentes da produção em mandioca. Pesq. Agropec. Bras., v.42, n. 8, p. 1121-1130, 2007.

OTSUBO, A. A. et al. Avaliação de clones de mandioca visando o processamento industrial em Dourados, MS. Dourados: 2007.

PEIXOTO, C. P. A mandioca. In: CASTRO, P. R. C.; KLUGE, $R$. A. Ecofisiologia de cultivos anuais: trigo, milho, soja, arroz e mandioca. Klunge. São Paulo: Nobel, 1999. p. 109-125.
PEIXOTO, J. R. et al. Desempenho agronômico de variedades de mandioca mansa em Uberlândia, MG. R. Bras. Mandioca, v. 18, n. 1, p. 19-24, 2005.

PITELLI, R. A. Interferência de plantas daninhas em cultivos agrícolas. Inf. Agropec., v. 11, n. 129, p. 16-26, 1985.

RIBEIRO Jr., J. I. Análises estatísticas no SAEG - guia prático. Viçosa, MG: Universidade Federal de Viçosa, 2004.

SANGOI, L.; KRUSE, N. D. Acúmulo e distribuição de matéria seca em diferentes frações da planta de mandioca no planalto catarinense. Pesq. Agropec. Bras., v. 28, n. 10, p. 1151-1164, 1993. 